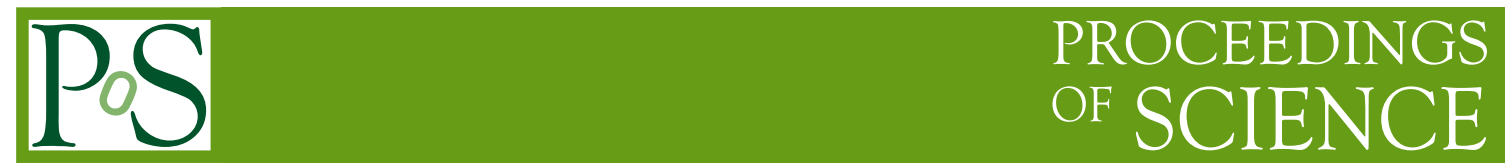

\title{
Transverse (Spin) Structure of Hadrons
}

\section{Matthias Burkardt*}

New Mexico State University

E-mail: burkardtenmsu.edu

Parton distributions in impact parameter space, which are obtained by Fourier transforming GPDs, exhibit a significant deviation from axial symmetry when the target and/or quark are transversely polarized. Connections between this deformation and transverse single-spin asymmetries as well as with quark-gluon correlations are discussed. The sign of transverse deformation of impact parameter dependent parton distributions in a transversely polarized target can be related to the sign of the contribution from that quark flavor to the nucleon anomalous magnetic moment. Therefore, the signs of the Sivers function for $u$ and $d$ quarks, as well as the signs of quark-gluon correlations embodied in the polarized structure function $g_{2}$ can be understood in terms of the proton and neutron anomalous magnetic moments.

Light Cone 2010 - LC2010

June 14-18, 2010

Valencia, Spain

${ }^{*}$ Speaker. 

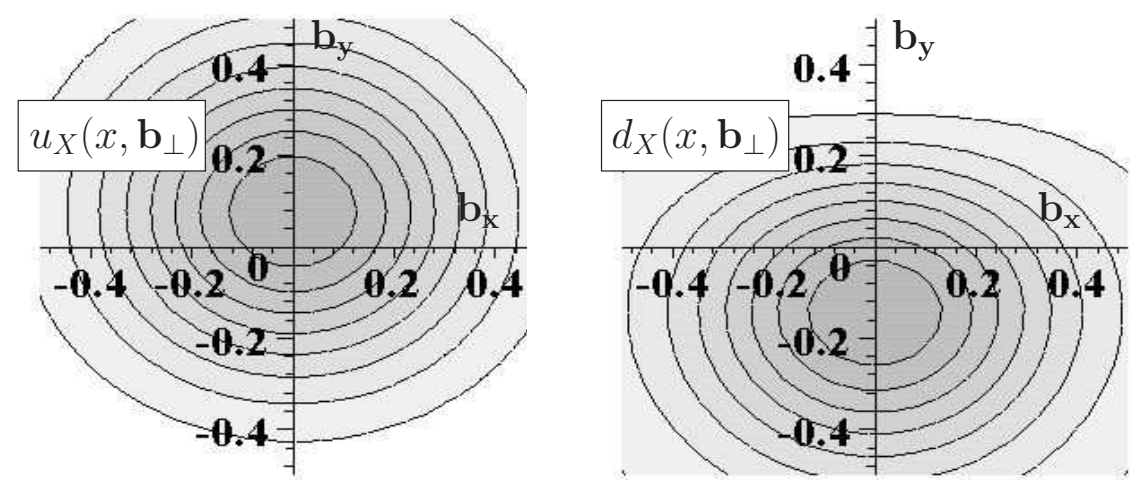

Figure 1: Distribution of the $j^{+}$density for $u$ and $d$ quarks in the $\perp$ plane $\left(x_{B j}=0.3\right)$ for a proton polarized in the $x$ direction in the model from Ref. [2]. For other values of $x$ the distortion looks similar. The signs of the distortion are determined by the signs of the contribution from each quark flavor to the proton anomalous magnetic moment.

\section{Impact Parameter Dependent Parton Distributions}

Generalized Parton Distributions (GPDs) can be obtained from the same light-cone wave function overlap integrals that yield form factors, except that the momentum fraction $x$ of the active quark is not integrated over, i.e. GPDs can be understood as an $x$ decomposition of form factors. The 2-dimensional Fourier transform of the GPD $H_{q}(x, 0, t)$ yields the distribution $q\left(x, \mathbf{b}_{\perp}\right)$ of unpolarized quarks and target, in impact parameter space [1]

$$
q\left(x, \mathbf{b}_{\perp}\right)=\int \frac{d^{2} \Delta_{\perp}}{(2 \pi)^{2}} H_{q}\left(x, 0,-\Delta_{\perp}^{2}\right) e^{-i \mathbf{b}_{\perp} \cdot \Delta_{\perp}}
$$

with $\Delta_{\perp}=\mathbf{p}_{\perp}^{\prime}-\mathbf{p}_{\perp}$. For a transversely polarized target (e.g. polarized in the $+\hat{x}$-direction) the impact parameter dependent PDF $q_{+\hat{x}}\left(x, \mathbf{b}_{\perp}\right)$ is no longer axially symmetric and the transverse deformation is described by the gradient of the Fourier transform of the GPD $E_{q}(x, 0, t)$ [2]

$$
q_{+\hat{x}}\left(x, \mathbf{b}_{\perp}\right)=q\left(x, \mathbf{b}_{\perp}\right)-\frac{1}{2 M} \frac{\partial}{\partial b_{y}} \int \frac{d^{2} \Delta_{\perp}}{(2 \pi)^{2}} E_{q}\left(x, 0,-\Delta_{\perp}^{2}\right) e^{-i \mathbf{b}_{\perp} \cdot \Delta_{\perp}}
$$

$E_{q}(x, 0, t)$ and hence the details of this deformation are not very well known, but its $x$-integral, the Pauli form factor $F_{2}$, is. Eq. (1.2) allows to relate the average transverse deformation

$$
d_{y}^{q} \equiv \int d x \int d^{2} \mathbf{b}_{\perp} q\left(x, \mathbf{b}_{\perp}\right) b_{y}=\frac{1}{2 M} \int d x E_{q}(x, 0,0)=\frac{\kappa_{q}^{p}}{2 M}
$$

to the contribution from the corresponding quark flavor to the anomalous magnetic moment

$\kappa_{u}^{p}=2 \kappa_{p}+\kappa_{n}=2 * 1.793-1.913=1.673$ and $\kappa_{d}^{p}=2 \kappa_{n}+\kappa_{p}=2 *(-1.913)+1.793=-2.033$. Since $\frac{1}{2 M} \approx 0.1 \mathrm{fm}$ this implies a very significant deformation $\left|d_{q}^{y}\right|=\mathscr{O}(0.2 \mathrm{fm})$ for both $u$ and $d$ quarks and in opposite directions.

For example, $u$ quarks in a proton contribute with a positive anomalous magnetic moment and $d$ quarks (after factoring out the negative $d$ quark charge) with a negative value. Eq. (1.2) thus implies that for a nucleon target polarized in the $+\hat{x}$ direction, the leading twist distribution of $u$ quarks is shifted in the $+\hat{y}$ direction while that of $d$ quarks is shifted in the $-\hat{y}$ direction (Fig. 1). This has important implications for the sign of transverse single-spin asymmetries (SSAs). 


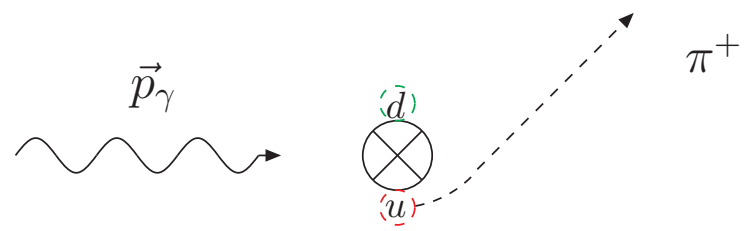

Figure 2: The transverse distortion of the parton cloud for a proton that is polarized into the plane, in combination with attractive FSI, gives rise to a Sivers effect for $u(d)$ quarks with a $\perp$ momentum that is on the average up (down).

\section{Transverse Single-Spin Asymmetries}

In a target that is polarized transversely (e.g. vertically), the quarks in the target can exhibit a (left/right) asymmetry of the distribution $f_{q / p^{\uparrow}}\left(x_{\mathrm{B}}, \mathbf{k}_{T}\right)$ in their transverse momentum $\mathbf{k}_{T}[3,4]$

$$
f_{q / p^{\uparrow}}\left(x_{\mathrm{B}}, \mathbf{k}_{T}\right)=f_{1}^{q}\left(x_{\mathrm{B}}, k_{T}^{2}\right)-f_{1 T}^{\perp q}\left(x_{\mathrm{B}}, k_{T}^{2}\right) \frac{\left(\hat{\mathbf{P}} \times \mathbf{k}_{T}\right) \cdot \mathbf{S}}{M},
$$

where $\mathbf{S}$ is the spin of the target nucleon and $\hat{\mathbf{P}}$ is a unit vector opposite to the direction of the virtual photon momentum. The fact that such a term may be present in (2.1) is known as the Sivers effect and the function $f_{1 T}^{\perp q}\left(x_{\mathrm{B}}, k_{T}^{2}\right)$ is known as the Sivers function. The latter vanishes in a naive parton picture since $\left(\hat{\mathbf{P}} \times \mathbf{k}_{T}\right) \cdot \mathbf{S}$ is odd under naive time reversal (a property known as naive-T-odd), where one merely reverses the direction of all momenta and spins without interchanging the initial and final states. The significant distortion of parton distributions in impact parameter space (Fig. 1) provides a natural mechanism for a Sivers effect. In semi-inclusive DIS, when the virtual photon strikes a $u$ quark in a $\perp$ polarized proton, the $u$ quark distribution is enhanced on the left side of the target (for a proton with spin pointing up when viewed from the virtual photon perspective). Although in general the final state interaction (FSI) is very complicated, we expect it to be on average attractive thus translating a position space distortion to the left into a momentum space asymmetry to the right and vice versa (Fig. 2) [5]. Since this picture is very intuitive, a few words of caution are in order. First of all, such a reasoning is strictly valid only in mean field models for the FSI as well as in simple spectator models [6]. Furthermore, even in such mean field or spectator models there is in general no one-to-one correspondence between quark distributions in impact parameter space and unintegrated parton densities (e.g. Sivers function) (for a recent overview, see Ref. [7]). While both are connected by an overarching Wigner distribution [8], they are not Fourier transforms of each other. Nevertheless, since the primordial momentum distribution of the quarks (without FSI) must be symmetric, we find a qualitative connection between the primordial position space asymmetry and the momentum space asymmetry due to the FSI. Another issue concerns the $x$-dependence of the Sivers function. The $x$-dependence of the position space asymmetry is described by the GPD $E\left(x, 0,-\Delta_{\perp}^{2}\right)$. Therefore, within the above mechanism, the $x$ dependence of the Sivers function should be related to that of $E\left(x, 0,-\Delta_{\perp}^{2}\right)$. However, the $x$ dependence of $E$ is not known yet and we only know the Pauli form factor $F_{2}=\int \mathrm{d} x E$. Nevertheless, if one makes the additional assumption that $E$ does not fluctuate as a function of $x$ then the contribution from each quark flavor $q$ to the anomalous magnetic moment $\kappa$ determines the sign of $E^{q}(x, 0,0)$ and hence of the Sivers function. With these assumptions, as well as the very plausible assumption that the FSI 
is on average attractive, one finds that $f_{1 T}^{\perp u}<0$, while $f_{1 T}^{\perp d}>0$. Both signs have been confirmed by a flavor analysis based on pions produced in a SIDIS experiment by the HERMES collaboration [9] and are consistent with a vanishing isoscalar Sivers function observed by COMPASS [10].

\section{Transverse Force on Quarks in DIS}

The chirally-even spin-dependent twist-3 parton distribution $g_{2}(x)=g_{T}(x)-g_{1}(x)$ is defined as

$$
\begin{aligned}
& \int \frac{d \lambda}{2 \pi} e^{i \lambda x}\left\langle P S\left|\bar{\psi}(0) \gamma^{\mu} \gamma_{5} \psi(\lambda n)\right|_{Q^{2}} \mid P S\right\rangle \\
& \quad=2\left[g_{1}\left(x, Q^{2}\right) p^{\mu}(S \cdot n)+g_{T}\left(x, Q^{2}\right) S_{\perp}^{\mu}+M^{2} g_{3}\left(x, Q^{2}\right) n^{\mu}(S \cdot n)\right]
\end{aligned}
$$

Neglecting $m_{q}$, one finds $g_{2}(x)=g_{2}^{W W}(x)+\bar{g}_{2}(x)$, with $g_{2}^{W W}(x)=-g_{1}(x)+\int_{x}^{1} \frac{d y}{y} g_{1}(y)$ [11], where $\bar{g}_{2}(x)$ involves quark-gluon correlations, e.g. [12, 13]

$$
\int d x x^{2} \bar{g}_{2}(x)=\frac{d_{2}}{3}
$$

with

$$
4 M P^{+} P^{+} S^{x} d_{2}=g\left\langle P, S\left|\bar{q}(0) G^{+y}(0) \gamma^{+} q(0)\right| P, S\right\rangle .
$$

At low $Q^{2}, g_{2}$ has the physical interpretation of a spin polarizability, which is why the matrix elements (note that $\sqrt{2} G^{+y}=B^{x}-E^{y}$ )

$$
\chi_{E} 2 M^{2} \vec{S}=\left\langle P, S\left|q^{\dagger} \vec{\alpha} \times g \vec{E} q\right| P, S\right\rangle \quad \chi_{B} 2 M^{2} \vec{S}=\left\langle P, S\left|q^{\dagger} g \vec{B} q\right| P, S\right\rangle
$$

are sometimes called spin polarizabilities or color electric and magnetic polarizabilities [14]. In the following we will discuss that at high $Q^{2}$ a better interpretation for these matrix elements is that of an average 'color Lorentz force' [15].

To see this we express the $\hat{y}$-component of the Lorentz force acting on a particle with charge $g$ that is moving with (nearly) the speed of light $\vec{v}=(0,0,-1)$ along the $-\hat{z}$ direction in terms of light-cone variables, yielding

$$
g[\vec{E}+\vec{v} \times \vec{B}]^{y}=g\left(E^{y}+B^{x}\right)=g \sqrt{2} G^{y+},
$$

which coincides with the component that appears in the twist-3 correlator above (3.2). Thus Eq. (3.2) represents the (twist 2) quark density correlated with the transverse color-Lorentz force that a quark would experience at that position if it moves with the velocity of light in the $-\hat{z}$ direction - which is exactly what the struck quark does after it has absorbed the virtual photon in a DIS experiment in the Bjorken limit. Therefore the correct semi-classical interpretation of Eq. (3.2) is that of an average ${ }^{1}$ transverse force

$$
\begin{aligned}
F^{y}(0) & \equiv-\frac{\sqrt{2}}{2 P^{+}}\left\langle P, S\left|\bar{q}(0) G^{+y}(0) \gamma^{+} q(0)\right| P, S\right\rangle \\
& =-2 \sqrt{2} M P^{+} S^{x} d_{2}=-2 M^{2} d_{2}
\end{aligned}
$$

\footnotetext{
${ }^{1}$ The average is meant as an ensemble average since the forward matrix element in plane wave states automatically provides an average over the nucleon volume.
} 
acting on the active quark in the instant right after ${ }^{2}$ it has been struck by the virtual photon.

Although the identification of $\left\langle p\left|\bar{q} \gamma^{+} G^{+y} q\right| p\right\rangle$ as an average color Lorentz force due to the final state interactions (3.5) may be intuitively evident from the above discussion, it is also instructive to provide a more formal justification. For this purpose, we consider the time dependence of the transverse momentum of the 'good' component of the quark fields (the component relevant for DIS in the Bjorken limit) $q_{+} \equiv \frac{1}{2} \gamma^{-} \gamma^{+} q$

$$
\begin{aligned}
2 p^{+} \frac{d}{d t}\left\langle p^{y}\right\rangle \equiv & \frac{d}{d t}\left\langle P S\left|\bar{q} \gamma^{+}\left(p^{y}-g A^{y}\right) q\right| P S\right\rangle \\
= & \frac{1}{\sqrt{2}} \frac{d}{d t}\left\langle P S\left|q_{+}^{\dagger}\left(p^{y}-g A^{y}\right) q_{+}\right| P S\right\rangle \\
= & 2 p^{+}\left\langle P S\left|\left[\dot{\bar{q}} \gamma^{+}\left(p^{y}-g A^{y}\right) q+\bar{q} \gamma^{+}\left(p^{y}-g A^{y}\right) \dot{q}\right]\right| P S\right\rangle \\
& -\left\langle P S\left|\bar{q} \gamma^{+} g \dot{A}^{y} q\right| P S\right\rangle .
\end{aligned}
$$

Using the QCD equations of motion

$$
\dot{q}=\left(i g A^{0}+\gamma^{0} \vec{\gamma} \cdot \vec{D}\right) q
$$

where $-i D^{\mu}=p^{\mu}-g A^{\mu}$, yields

$$
\begin{aligned}
2 p^{+} \frac{d}{d t}\left\langle\mathbf{p}^{y}\right\rangle & =\left\langle P S\left|\bar{q} \gamma^{+} g\left(G^{y 0}+G^{y z}\right) q\right| P S\right\rangle+{ }^{'}\left\langle P S\left|\bar{q} \gamma^{+} \gamma^{-} \gamma^{i} D^{i} D^{j} q\right| P S\right\rangle^{\prime} \\
& =\sqrt{2}\left\langle P S\left|\bar{q} \gamma^{+} g G^{y+} q\right| P S\right\rangle+{ }^{\prime}\left\langle P S\left|\bar{q} \gamma^{+} \gamma^{-} \gamma^{i} D^{i} D^{j} q\right| P S\right\rangle^{\prime},
\end{aligned}
$$

where ' $\left\langle P S\left|\bar{q} \gamma^{+} \gamma^{-} \gamma^{i} D^{i} D^{j} q\right| P S\right\rangle^{\prime}$ stands symbolically for all terms that involve a product of $\gamma^{+} \gamma^{-}$ as well as a $\gamma^{\perp}$ and only $\perp$ derivatives $D^{i}$.

Now it is important to keep in mind that we are not interested in the average force on the 'original' quark fields (before the quark is struck by the virtual photon), but after absorbing the virtual photon and moving with (nearly) the speed of light in the $-\hat{z}$ direction. In this limit, the first term on the r.h.s. of (3.8) dominates, as it contains the largest number of ' + ' Lorentz indices. Dropping the other terms yields (3.5).

The identification of $2 M^{2} d_{2}$ with the average transverse force acting on the active quark in a SIDIS experiment is also consistent with the Qiu Sterman result [16] for the average transverse momentum of the ejected quark (also averaged over the momentum fraction $x$ carried by the active quark)

$$
\left\langle k_{\perp}^{y}\right\rangle=-\frac{1}{2 P^{+}}\left\langle P, S\left|\bar{q}(0) \int_{0}^{\infty} d x^{-} G^{+y}\left(x^{+}=0, x^{-}\right) \gamma^{+} q(0)\right| P, S\right\rangle
$$

The average transverse momentum is obtained by integrating the transverse component of the color Lorentz force along the trajectory of the active quark - which is an almost light-like trajectory along the $-\hat{z}$ direction, with $z=-t$. The local twist-3 matrix element describing the force at time $=0$ is the first integration point in the Qiu-Sterman integral (3.9).

Lattice calculations of the twist-3 matrix element yield [17]

$$
d_{2}^{(u)}=0.010 \pm 0.012 \quad d_{2}^{(d)}=-0.0056 \pm 0.0050
$$

\footnotetext{
${ }^{2}$ 'Right after', since the quark-gluon correlator in (3.5) is local!
} 
renormalized at a scale of $Q^{2}=5 \mathrm{GeV}^{2}$ for the smallest lattice spacing in Ref. [17]. These numbers are also consistent with experimental studies [18]. Using (3.5) these (ancient) lattice results thus imply

$$
F_{(u)} \approx-100 \mathrm{MeV} / \mathrm{fm} \quad F_{(d)} \approx 56 \mathrm{MeV} / \mathrm{fm} .
$$

In the chromodynamic lensing picture, one would have expected that $F_{(u)}$ and $F_{(d)}$ are of about the same magnitude and with opposite sign. The same holds in the large $N_{C}$ limit. A vanishing Sivers effect for an isoscalar target would be more consistent with equal and opposite average forces. However, since the error bars for $d_{2}$ include only statistical errors, the lattice result may not be inconsistent with $d_{2}^{(d)} \sim-d_{2}^{(u)}$.

The average transverse momentum from the Sivers effect is obtained by integrating the transverse force to infinity (along a light-like trajectory) $\left\langle k^{y}\right\rangle=\int_{0}^{\infty} d t F^{y}(t)$. This motivates us to define an 'effective range'

$$
R_{e f f} \equiv \frac{\left\langle k^{y}\right\rangle}{F^{y}(0)}
$$

Note that $R_{\text {eff }}$ depends on how rapidly the correlations fall off along a light-like direction and it may thus be larger than the (spacelike) radius of a hadron. Of course, unless the functional form of the integrand is known, $R_{\text {eff }}$ cannot really tell us about the range of the FSI, but if the integrand in (3.5) does not oscillate, (3.12) provides a reasonable estimate for the range over which the integrand in (3.5) is significantly nonzero.

Fits of the Sivers function to SIDIS data yield about $\left|\left\langle k^{y}\right\rangle\right| \sim 100 \mathrm{MeV}$ [19]. Together with the (average) value for $\left|d_{2}\right|$ from the lattice this translates into an effective range $R_{e f f}$ of about $1 \mathrm{fm}$. It would be interesting to compare $R_{\text {eff }}$ for different quark flavors and as a function of $Q^{2}$, but this requires more precise values for $d_{2}$ as well as the Sivers function.

A relation similar to (3.5) can be derived for the $x^{2}$ moment of the twist-3 scalar PDF $e(x)$. For its interaction dependent twist-3 part $\bar{e}(x)$ one finds for an unpolarized target [20]

$$
4 M P^{+} P^{+} e_{2}=g\left\langle p\left|\bar{q} \sigma^{+i} G^{+i} q\right| P\right\rangle,
$$

where $e_{2} \equiv \int_{0}^{1} d x x^{2} \bar{e}(x)$. The matrix element on the r.h.s. of Eq. (3.13) can be related to the average transverse force acting on a transversely polarized quark in an unpolarized target right after being struck by the virtual photon. Indeed, for the average transverse momentum in the $+\hat{y}$ direction, for a quark polarized in the $+\hat{x}$ direction, one finds

$$
\left\langle k^{y}\right\rangle=\frac{1}{4 P^{+}} \int_{0}^{\infty} d x^{-} g\left\langle p\left|\bar{q}(0) \sigma^{+y} G^{+y}\left(x^{-}\right) q(0)\right| p\right\rangle .
$$

A comparison with Eq. (3.13) shows that the average transverse force at $t=0$ (right after being struck) on a quark polarized in the $+\hat{x}$ direction reads

$$
F^{y}(0)=\frac{1}{2 \sqrt{2} p^{+}} g\left\langle p\left|\bar{q} \sigma^{+y} G^{+y} q\right| p\right\rangle=\frac{1}{\sqrt{2}} M P^{+} S^{x} e_{2}=\frac{M^{2}}{2} e_{2} .
$$

The impact parameter distribution for quarks polarized in the $+\hat{x}$ direction [21] is shifted in the $+\hat{y}$ direction $[22,23]$. Applying the chromodynamic lensing mechanism implies a force in 
the negative $\hat{y}$ direction for these quarks and one thus expects $e_{2}<0$ for both $u$ and $d$ quarks. Furthermore, since $\kappa_{\perp}>\kappa$, one would expect that in a SIDIS experiment the $\perp$ force on a $\perp$ polarized quark in an unpolarized target on average to be larger than that on unpolarized quarks in a $\perp$ polarized target, and thus $\left|e_{2}\right|>\left|d_{2}\right|$.

\section{Summary}

The GPD $E^{q}\left(x, 0,-\Delta_{\perp}^{2}\right)$, which arises in the ' $x$-decomposition' of the contribution from quark flavor $q$ to the Pauli form factor $F_{2}^{q}$ describes the transverse deformation of the unpolarized quark distribution in impact parameter space. That deformation provides a very intuitive mechanism for transverse SSAs in SIDIS. As a result, the signs of SSAs can be related to the contribution from quark flavor $q$ to the nucleon anomalous magnetic moment. Quark-gluon correlations appearing in the $x^{2}$-moment of the twist-3 part of the polarized parton distribution $g_{2}^{q}(x)$ have a semi-classical interpretation as the average (enemble average) transverse force acting on the struck quark in DIS from a transversely polarized target in the moment after it has absorbed the virtual photon. Since the direction of that force can be related to the transverse deformation of PDFs, one can thus also relate the sign of these quark-gluon correlations to the contribution from quark flavor $q$ to the nucleon anomalous magnetic moment.

Such a correlation between observables that at first appear to have little in common also occurs in the chirally odd sector: the impact parameter space distribution of quarks with a given transversity in an unpolarized target can be related to the Boer-Mulders function describing the left-right asymmetry of quarks with a given transversity in SIDIS from an unpolarized target. Furthermore, semi-classically, the quark-gluon correlations appearing in the $x^{2}$-moment of the twist-3 part of the scalar PDF $e(x)$ describes the average transverse force acting on a quark with given transversity immediately after it has absorbed the virtual photon.

Acknowledgements: I would like to thank A.Bacchetta, D. Boer, J.P. Chen, Y.Koike, and Z.-E. Mezziani for useful discussions. This work was supported by the DOE under grant number DE-FG03-95ER40965.

\section{References}

[1] M.Burkardt, Phys. Rev. D 62, 071503 (2000), Erratum-ibid. D 66, 119903 (2002); M. Diehl, Eur. Phys. J. C 25, 223 (2002); J.P. Ralston and B. Pire, Phys. Rev. D 66, 111501 (2002).

[2] M. Burkardt, Int. J. Mod. Phys. A 18, 173 (2003).

[3] D.W. Sivers, Phys. Rev. D 43, 261 (1991).

[4] A. Bacchetta et al., Phys. Rev. D 70, 117504 (2004).

[5] M. Burkardt, Phys. Rev. D 66, 114005 (2002); Phys. Rev. D 69, 057501 (2004).

[6] S.J. Brodsky, D.S. Hwang, and I. Schmidt, Nucl. Phys. B 642, 344 (2002); M. Burkardt and D.S. Hwang, Phys. Rev. D69, 074032 (2004); L.P. Gamberg et al., Phys. Rev. D 67, 071504 (2003); D. Boer, S.J. Brodsky, and D.S. Hwang, Phys. Rev. D 67, 054003 (2003); A. Bacchetta et al., Phys. Lett B578, 109 (2004); M. Radici et al., hep-ph/0708.0232; L.P. Gamberg et al. hep-ph/0708.0324; D. Boer et al., Phys. Rev. D 67, 054003 (2003); L.P. Gamberg et al., Phys. Rev. D 67 (2003) 071504. 
[7] S. Meissner et al., Phys. Rev. D 76, 034002 (2007)

[8] A.V. Belitsky, X. Ji, and F. Yuan, Phys. Rev. D 69, 074014 (2004).

[9] A. Airapetian et al. (Hermes collab.), Phys. Rev. Lett. 94, 012002 (2005).

[10] A. Martin (Compass collab.), Czech. J. Phys. 56, F33 (2006).

[11] S. Wandzura and F. Wilczek, Phys. Lett. 72B, 195 (1977).

[12] E. Shuryak and A.I. Vainshtein, Nucl. Phys. B 201, 141 (1982).

[13] R.L. Jaffe, Comm. Nucl. Part. Phys. 19, 239 (1990).

[14] B.W. Filippone and X. Ji, Adv. Nucl. Phys. 26, 1 (2001).

[15] M. Burkardt, arXiv:0811.1206

[16] J. Qiu and G. Sterman, Phys. Rev. Lett. 67, 2264 (1991).

[17] M. Göckeler et al., Phys. Rev. D 72, 054507 (2005).

[18] Z.-E. Mezziani et al., hep-ph/0404066.

[19] M. Anselmino et al., Eur. Phys. J. A 39, 89 (2009).

[20] Y. Koike and K. Tanaka, Phys. Rev. D 51, 6125 (1995).

[21] M. Diehl and P.Hägler, Eur. Phys. J. C44, 87 (2005).

[22] M. Göckeler et al. (QCDSF collaboration), Phys. Rev. Lett. 98, 222001 (2007); Ph. Hägler et al. (LHPC collaboration), hep-lat/0705.4295.

[23] M. Burkardt and B. Hannafious, Phys. Lett. B 658, 130 (2008). 